\title{
Tratamento das Uropatias Obstrutivas Fetais: Experiência com Novo Cateter
}

\author{
A New Catheter in the Treatment of fetal Obstructive Uropathies
}

Ana Paula B.M. Lopes, Antônio Carlos V. Cabral, Henrique Vitor Leite

\section{Resumo}

A abordagem das uropatias diagnosticadas no periodo pré-natal é controversa, principalmente, porque o prognóstico desses fetos é variável. Contudo, trabalhos pioneiros têm demonstrado que a drenagem pré-natal do trato urinário obstruido pode melhorar o resultado em fetos selecionados. O objetivo deste trabalho é relatar a experiência do Serviço no tratamento das lesões obstrutivas do trato urinário, utilizando o cateter desenvolvido no Centro de Medicina Fetal do Hospital das Clínicas da UFMG. No total, 25/25 fetos com uropatia obstrutiva receberam o cateter. Três fetos necessitaram de mais de uma inserção; 10 dos 25 fetos que receberam o cateter (40\%) sobreviveram com boa função renal e pulmonar pós-natal. Ocorreram complicações em 12/25 casos (48\%) incluindo 6 com drenagem inadequada ou migração do cateter (24\%); 1 / 25 (4\%) ascite urinária; $1 / 25$ (4\%) descolamento prematuro de placenta; $1 / 25$ (4\%) rutura prematura de membrana; $2 / 25$ (08\%) trabalho de parto prematuro; 01/25 (04\%) fibrose ou cicatrização do parênquima renal. Três dos 25 fetos (12\%) morreram intra-útero e 12 (48\%) fetos morreram no periodo neonatal. Concluindo, a drenagem do trato urinário com esse cateter provou ser tecnicamente possível e segura para mãe e feto, com uma taxa de sobrevida de $40 \%$.

PALAVRAS-CHAVE: Malformações fetais. Malformações urogenitais. Uropatia. Terapia fetal.

As lesões obstrutivas do trato urinário fetal envolvem um grupo heterogêneo de anomalias do desenvolvimento que resultam em obstrução parcial ou completa do fluxo de urina em algum nivel do trato urinário ${ }^{12}$. Correspondem a $17 \%$ das anomalias fetais diagnosticadas intra-útero, estando presentes na proporção de 1:200 a 1:1000 nascimentos. Durante o desenvolvimento do sistema geniturinário fetal a obstrução pode ocorrer em três diferentes níveis: 1) junção ureteropélvica, 2) junção ureterovesical, 3) obstrução uretral

Centro de Medicina Fetal-Hospital das Clínicas da Universidade Federal de Minas Gerais.

Correspondência:

Ana Paula Brum M. Lopes

R. Wilson Modesto Ribeiro, 55, ap. 404 - cep: 31160-430

Belo Horizonte - MG sendo, desse modo, responsável por um amplo espectro de acometimento fetal, causando, nos casos graves, seqüelas renais e pulmonares irreversiveis que inviabilizam a sobrevida neonatal.

Nas últimas décadas grande tem sido a evolução da abordagem pré-natal dessas lesões fetais. Os estudos em animais realizados por Harrison e colaboradores na década de 80 em muito contribuíram para o esclarecimento da história natural e fisiopatologia dessa doença fetal. O grande avanço conseguido por esses mesmos autores foi, no entanto, o desenvolvimento de um cateter de derivação urinária, destinado ao tratamento de tais lesões intra-útero e que se mostrou útil em drenar o sistema obstruído e restaurar o volume de líquido amniótico melhorando o prognóstico fetal ${ }^{7}$. 
A seleção correta dos casos destinados a receber o tratamento tem tornado os resultados progressivamente melhores ${ }^{6}$.

Em nosso Serviço, temos priorizado não apenas a terapêutica fetal convencional, mas também buscado a melhoria da qualidade de sobrevida neonatal procurando adaptar os avanços científicos à nossa realidade e ao nosso ambiente de trabalho. Com esse objetivo foi desenvolvido por profissionais do Serviço um cateter de polietileno do tipo double pig-tail ${ }^{1}$ que possui as vantagens de ser menos calibroso, mais flexivel, de mais fácil aquisição e mais econômico que o cateter original descrito por Golbus et al. ${ }^{7}$. O objetivo desse trabalho foi, portanto, descrever a experincia do nosso Serviço no tratamento das lesões obstrutivas do trato urinário fetal com a utilização do referido cateter, avaliando não apenas as dificuldades e complicações materno-fetais relacionadas ao procedimento, como também sua eficácia em promover a descompressão do trato urinário e aliviar as seqüelas fetais da uropatia obstrutiva.

\section{Pacientes e Métodos}

Durante o período de janeiro de 1993 a dezembro de 1996, foram selecionadas para participarem do estudo 25 gestantes, apresentando gestações simples, encaminhadas ao Serviço de Pré-Natal do Centro de Medicina Fetal por apresentarem diagnóstico ultra-sonográfico de lesões obstrutivas do trato urinário fetal. Todas as pacientes que procuravam o Serviço com suspeita de uropatias destrutivas eram submetidas a exame ultra-sonográfico pela mesma equipe de examinadores, utilizando-se equipamento de ultrasom de tempo real, Schimatzu (Shimasonic SDL 300), e transdutor convexo de $3,5 \mathrm{mHz}$, com a finalidade de determinar o nível de obstrução e a confirmação do diagnóstico.

A seleção dos fetos que seriam submetidos à descompressão intra-uterina do trato urinário, utilizando-se o cateter em estudo, foi realizada de acordo com os seguintes critérios seguindo-se o protocolo do Serviço:

1 - idade gestacional inferior a 33 semanas completas (determinada por datamento cronológico e/ou exame ultra-sonográfico realizado até 20 semanas);

2 - presença de lesão obstrutiva bilateral causando hidronefrose bilateral progressiva;

3- presença de lesão unilateral que pelo seu volume acentuado esteja comprimindo o rim contralateral e causando hidronefrose do mesmo;
4- ausência de outras anomalias fetais que limitem a vida extra-uterina e/ou carióipo normal;

5- presença de volume do líquido amniótico diminuído. Foi utilizado o índice de líquido amniótico proposto por Phelan et al. ${ }^{14}$, considerando-se como oligoâmnio valores abaixo de $8 \mathrm{~cm}$;

6- ausência de sinais sugestivos de comprometimento da função renal. Para tanto consideramos os parâmetros ultra-sonográficos: aspecto e ecogenicidade do parênquima renal normal e ausência de cistos corticais. O parâmetro bioquímico empregado: foi a determinação da osmolaridade da urina fetal obtida por punção da bexiga ou rim considerando-se a função renal preservada com valores $<210 \mathrm{mOsm} / \mathrm{ml}$.

Utilizamos um cateter do tipo double pig tail de polietileno medindo $3 \mathrm{~cm}$ de comprimento e 3 mm de diâmetro, sendo composto de duas espirais de duas voltas e meia cada uma separadas por um segmento retilineo (Figura 1).

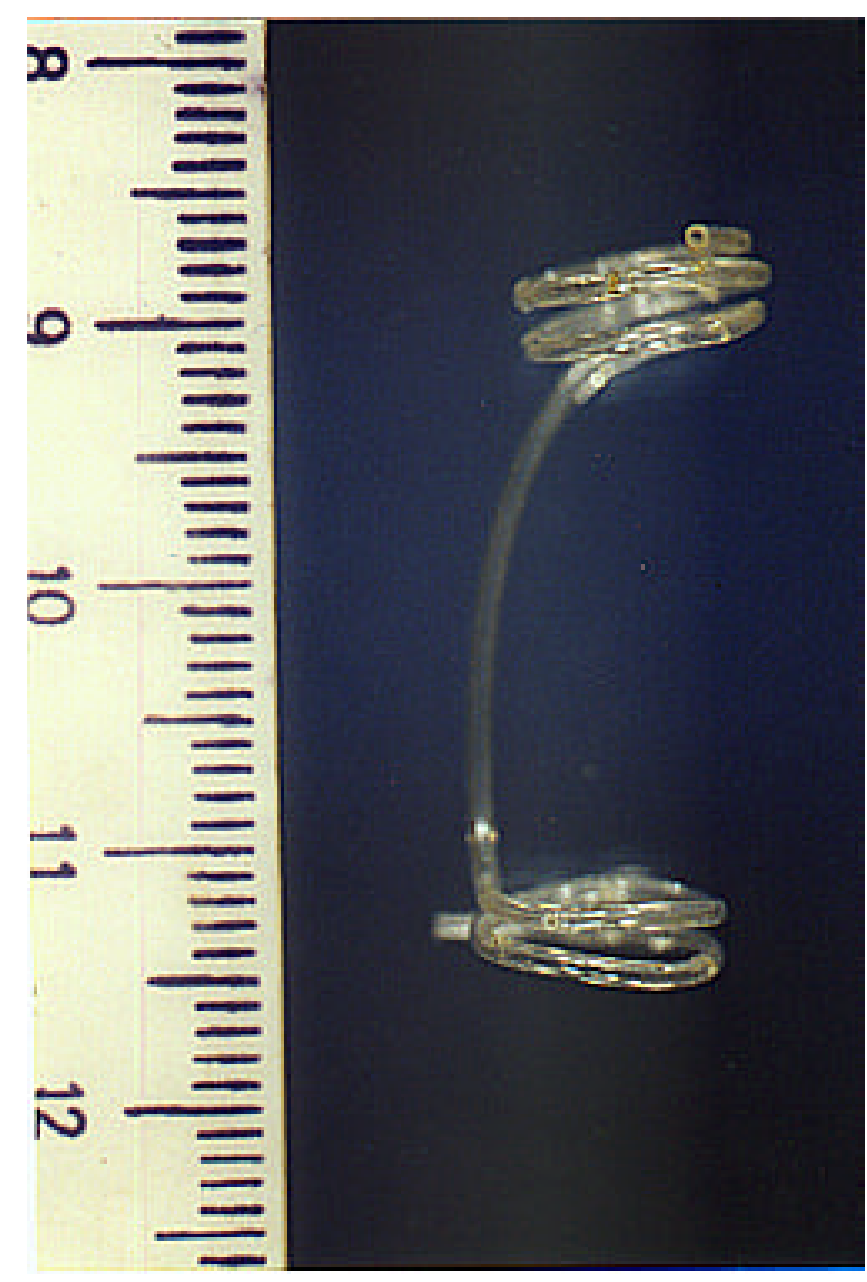

Figura 1 - Cateter de derivação do trato urinário fetal ${ }^{1}$ 
O procedimento consiste na colocação intrauterina do cateter a juzante do processo obstrutivo, guiado por visão ultra-sonográfica, após rigorosa anti-sepsia da pele. Foi realizada anestesia local na parede do abdome materno com lidocaína $0,1 \%$, sendo a introdução do cateter realizada com auxílio de um mandril após punção do abdome materno com agulha de Silvius calibre $16 \mathrm{~F}$. Ao atingir o local desejado (pelve renal ou bexiga fetal) o cateter era retirado da agulha com a ajuda do mandril, permanecendo uma extremidade do mesmo na estrutura a ser drenada e outra extremidade na cavidade amniótica.

As pacientes submetidas ao procedimento eram avaliadas clínica e ultra-sonograficamente com intervalos semanais até a resolução da gestação. Foram consideradas complicações do tratamento intra-uterino aquelas que ocorreram no intervalo de até uma semana após o procedimento.

Pacientes que apresentavam oligoâmnio acentuado, dificultando o diagnóstico morfológico, foram submetidas a amnioinfusão. Pacientes que apresentavam outras anomalias associadas às lesões obstrutivas foram submetidas a análise do cariótipo. Os fetos que evoluíram para morte intrauterina assim como récem-nascidos que evoluíram para a morte neonatal foram submetidos ao estudo anatomopatológico.

Para determinação da incidência de complicações e de sucesso do procedimento nas diversas variáveis analisadas realizamos o cálculo porcentual.

\section{Resultados}

Nos 25 casos acompanhados durante o estudo foram realizadas 31 punções destinadas à inserção do cateter, resultando em uma média de 1,2 punções por paciente.

Em 21 dos 25 casos (84\%) o cateter foi inserido na primeira tentativa; em dois casos $(8 \%)$ foram necessárias duas punções para inserção do cateter e em apenas um caso (4\%) foram necessárias três tentativas.

A idade gestacional média quando da inserção do cateter foi de 26,1 semanas (com variação entre 18 e 33 semanas).

A idade gestacional média de diagnóstico das lesões obstrutivas do trato urinário foi de 25 semanas (com variação entre 17 e 32 semanas). O tempo médio de permanência do cateter da colocação até o parto foi de 46 dias.

O diagnóstico morfológico antenatal mais freqüentemente encontrado nos casos em estudo foi a obstrução uretral (56\%), seguido da obstrução da junção ureteropélvica (36\%), sendo a obstrução da junção ureterovesical diagnosticada em dois casos (8\%).

O procedimento mais utilizado foi a colocação da derivação vesicoamniótica (56\%) acompanhada pela derivação pieloamniótica (44\%).

Na Tabela 1 observamos que 13 dos 25 fetos (52\%) evoluíram sem complicações relacionadas ao procedimento. Dentre os fetos que apresentaram complicações a mais freqüentemente observada foi o deslocamento ou oclusão do cateter.

Tabela 1 - Distribuição das pacientes de acordo com a ocorrência de complicações relacionadas ao procedimento.

\begin{tabular}{lcc}
\multicolumn{1}{c}{ Complicações } & Freqüência & $\%$ \\
\hline $\begin{array}{l}\text { Descolamento prematuro de } \\
\text { placenta }\end{array}$ & 1 & 4 \\
$\begin{array}{l}\text { Rotura prematura de } \\
\text { membranas }\end{array}$ & 1 & 4 \\
Trabalho de parto prematuro & 2 & 8 \\
Ascite fetal & 1 & 4 \\
Fibrose do parênquima renal & 1 & 4 \\
$\begin{array}{l}\text { Reacúmulo de líquido no } \\
\text { trato urinário fetal }\end{array}$ & 6 & 24 \\
\hline Sem complicações & 13 & 52 \\
\hline TOTAL & 25 & 100
\end{tabular}

Três casos (12\%) evoluíram para óbito intrauterino, não sendo possivel identificar a causa mortis em nenhum desses fetos. Quatro neomortos apresentaram outras malformações associadas assim distribuídas: um com seqüência de Vater, um com síndrome de Fryns, um com trissomia do 21 e um caso com infecção por citomegalovírus. Dos casos que apresentaram sobrevida, 10 em 25 (40\%), não apresentaram comprometimento da função renal ou pulmonar no período de acompanhamento neonatal. (Tabela 2).

Tabela 2 - Evolução dos fetos submetidos ao tratamento intra-uterino com o cateter

\begin{tabular}{|c|c|c|}
\hline Resultado & Frequência & $\%$ \\
\hline Morte fetal & 3 & 12 \\
\hline Morte neonatal & 12 & 48 \\
\hline $\begin{array}{l}\text { * Morte neonatal } \\
\text { depurada }\end{array}$ & 8 & 32 \\
\hline Sobrevida neonatal & 10 & 40 \\
\hline
\end{tabular}


Das 25 gestações acompanhadas o oligoâmnio esteve presente em 23 (92\%), sendo que se conseguiu restaurar o volume de líquido amniótico em 18 gestações (78\%).

A hipoplasia pulmonar esteve presente em $52 \%$ dos casos (13/25), sendo responsável direta pela morte neonatal em 11 récem-nascidos $(91,6 \%$ dos óbitos). Em nove dos 13 fetos que apresentaram hipoplasia pulmonar o volume de líquido amniótico havia sido restaurado, um apresentou volume de líquido amniótico normal e em três casos não foi possível recuperar o volume de líquido amniótico.

Dois fetos por nós acompanhados apresentaram diagnóstico anatomopatológico de displasia renal.

\section{Discussão}

As lesões obstrutivas do trato urinário fetal são diagnosticadas com elevada freqüência no período antenatal pois, em sua maioria, resultam na formação de lesões císticas facilmente identificadas ao exame ultra-sonográfico. O diagnóstico pré-natal dessas lesões apresenta importante significado clínico uma vez que permitem alterar a conduta diante dos fetos acometidos. Nos casos devidamente selecionados, uma vez realizado o diagnóstico de lesão obstrutiva do trato urinário fetal, é necessária a intervenção antenatal pois a obstrução, quando não-aliviada, interfere com o desenvolvimento fetal sendo que a gravidade do acometimento varia de acordo com o grau e a duração da obstrução. No caso de não reconhecimento da lesão ou não realização do tratamento a sobrevida perinatal é esporádica e nesses casos a qualidade de vida pós nascimento é seriamente comprometida por quadros de insuficiência renal grave.

A correção dessas lesões intra-uterinas foi realizada primeiramente no inicio da década de 80 por Harrison et al. ${ }^{8}$.O principal objetivo dessa intervenção é descomprimir o trato urinário fetal e restaurar o volume do líquido amniótico melhorando o prognóstico fetal.

Em 21 dos casos (84\%) foi necessária apenas uma punção para inserção do cateter. Na literatura, no entanto, encontramos a necessidade de realização de um número maior de tentativas para inserção do cateter. O Registro Internacional de Cirurgia Fetal relata que, em apenas $8,2 \%$ dos fetos, o cateter é inserido na primeira tentativa ${ }^{13}$. Atribuímos esse fato ao menor calibre do cateter por nós utilizado.

A idade gestacional em que se fez o diagnóstico das uropatias obstrutivas do trato urinário, em nossa casuística, foi maior que aquele observado na literatura ( 25 semanas), ao passo que nesta encontramos uma média de 23 semanas $^{4-13}$.

A derivação urinária intra-uterina é um procedimento invasivo e como tal apresenta riscos materno-fetais. A taxa de complicações por nós encontrada não diferiu muito do citado na literatura ${ }^{5}$. Não encontramos em nosso estudo, no entanto, casos de corioamnionite, muito citados na literatura ${ }^{3-10}$ o que atribuímos à eficácia da antisepsia realizada.

O oligoâmnio esteve presente em $92 \%$ das gestações estudadas, mais freqüente que o encontrado na literatura: 49 a $78 \%^{2,5,11}$. A maior freqüência de oligoâmnio nesse estudo pode ser devida ao fato de ser um dos critérios de inclusão no estudo e ao fato desse ser um achado comum em associação com as obstruções uretrais às quais corresponderam a maior parte dos nossos diagnósticos.

A recuperação do volume do líquido amniótico após a derivação ocorreu em $78 \%$ dos casos de oligoâmnio. Dentre os cinco casos nos quais não ocorreu recuperação do volume do líquido amniótico dois apresentavam rins nãofuncionantes ao exame anatomopatológico (um caso de rim multicístico bilateral e um caso de displasia renal bilateral); em um caso a extremidade amniótica do cateter drenou para cavidade peritonial do feto. Em dois casos foi necessária a resolução da gravidez algumas horas após a colocação do cateter (uma rutura prematrura de membrana e um descolamento prematuro de placenta). Esses achados demonstram que o cateter em estudo é eficaz em descomprimir o trato urinário fetal.

A hipoplasia pulmonar esteve presente em $52 \%$ dos casos acompanhados, não diferindo do relatado na literatura que demonstra alta freqüência de acometimento pulmonar em fetos portadores de uropatias obstrutivas ${ }^{12-13}$. A hipoplasia pulmonar foi encontrada inclusive em fetos que apresentaram recuperação do volume de líquido amniótico e em um caso no qual o líquido amniótico apresentou volume normal durante toda a gestação. Podemos verificar, então, que outros fatores além do volume de líquido amniótico devem contribuir para a gênese da hipoplasia pulmonar como idade gestacional de acometimento ou mesmo algum fator renal que atue no desenvolvimento pulmonar.

Encontramos apenas três casos de displasia renal confirmada nos fetos por nós acompanhados. Esses dois fetos tinham diagnóstico antenatal de válvula de uretra posterior com hidronefrose bilateral e função renal preservada constituindo então falha diagnóstica. 
A literatura relata uma taxa de neomortalidade que varia de 24,2 a $51 \%{ }^{4}$. Quando excluímos as mortes neonatais relacionadas a outras malformações encontramos uma taxa de morte neonatal de $32 \%$, situando-se dentro do limite citado.

A sobrevida neonatal encontrada foi de $40 \%$, muito próxima da encontrada por Harrison et al. ${ }^{10}$ que observaram taxas de $40 \%$ e Manning et al. ${ }^{13}$ que relatam sobrevida de $41 \%$.

Temos certeza de que a sobrevida de $40 \%$ obtida entre os fetos por nós tratados representa um número expressivo em uma situação patológica na qual a evolução para o óbito é a regra.

A nosso ver, a contribuíção deste estudo foi de apresentar um cateter de baixo custo e de confecção própria utilizado na derivação das lesões obstrutivas do feto, com os mesmos resultados relacionados na literatura mundial, onde são utilizados cateteres de elevado custo e pouca disponibilidade em nosso meio.

Consideramos que os resultados alcançados até o momento com a terapia intra-uterina para lesões obstrutivas representam um grande avanço. No entanto, muitas questões permanecem sem respostas. Outros estudos são ainda necessários para que o verdadeiro papel da terapia intra-uterina possa ser definitivamente estabelecido.

\section{SUMMARY}

Management of prenatally diagnosed uropathies is controversial, mainly because the prognosis for these fetuses is quite different. However pioneering studies have shown that prenatal drainage of obstructed urinary tract can improve the outcome of selected fetuses. The aim of this study is to describe the experience of the Service with the treatment of fetal obstrutive uropathy with the catheter developed by the Centro de Medicina Fetal do Hospital das Clínicas da UFMG. A total of 25 fetuses with obstructive uropathy received the catheter. Three fetuses required more than one insertion. Ten of $25(40 \%)$ shunted fetuses survived with good postnatal renal and pulmonary function. Complications occurred in 12/25 (48\%) cases including: 06/25 (24\%) inadequate shunt drainage or migration; 01/25 (04\%) urinary ascitis; 01/25 (04\%) DPP, 01/25 (04\%) premature rupture of membranes, $02 / 25(08 \%)$ premature labor, 01/25 (04\%) scarring and fibrosis of the renal parenchyma. Three of 25 (12\%) fetuses died intra-utero and 12 (48\%) died during the neonatal period. In conclusion, the drainage of the obstructed urinary tract with this catheter proved to be technically feasible and safe for both mother and fetus, with a survival rate of $40 \%$.

KEY WORDS: Obstructive uropathy. Fetal therapy. Fetal anomalies.

\section{Referências bibliográficas}

1. Cabral ACV, Leite HV, Auiar RLP, Oliveira EA. Tratamento da obstrução de uretra fetal. Relato de dois casos. J Bras Ginecol 1992; 102: 121-2.

2. Callan NA, Blakemore K, Park J, Sanders RC, Jeffs RD, Gearhart JP. Fetal genitourinary tract anomalies: evaluation, operative correction, and followup. Obstet Gynecol 1990; 75: 67-74.

3. Crombleholme TM, Harrison MR, Golbus MS, Longaker MT, Langer JC, Callen PW, Aanderson RL, Goldstein RB, Filly RA. Fetal intervention in obstructive uropathy: prognostic indicators and efficacy of intervention. Am J Obstet Gynecol 1990; 162: 1239-44.

4. Cusick EL, Didier F, Droulle P, Schmitt M. Mortality after an antenatal diagnosis of foetal uropathy. J Pediatr Surg 1995; 30: 463-6.

5. Eelder JS, Duckett JW, Snyder HM. Intervention for fetal obstructive uropathy: has it been effective? Lancet, 1987; 21: 1007-10.

6. Glick PL, Harrison MR, Golbus MS, Adzick NS, Filly RA, Callen PW, Mahony BS, Anderson RL, Lorimer AA. Management of the fetus with congenital hydronephrosis II. Prognostic criteria and selection for treatment. J Pediatr Surg 1985; 20: 376-87.

7. Golbus MS, Harrison MR, Filly RA, Callen PW, Katz, M. In utero treatment of urinary tract obstruction. Am J Obstet Gynecol 1982; 142: 383-8.

8. Harrison MR, Golbus MS, Filly RA, Nakayama DK, Callen PW, Lorimer AA, Hricak H. Management of the fetus with congenital hydronephrosis. J Pediatr Surg 1982; 17: 728-42.

9. Harrison MR, Ross N, Noal R. Correction of congenital hydronephrosis in utero. I: The model: Fetal uretral obstruction produces hydronephrosis and pulmonary hypoplasia in fetal lambs. J Pediatr Surg 1983; 18: 247-255

10.Harrison MR, Filly RA. The fetus with obstrutive uropathy: patophysiology natural history, selection and treatment. In: Harrison MR, Golbus MS, Filly RA editors. The unborn patient: prenatal diagnosis and treatment. $2^{\text {nd }}$ ed. Philadelphia: Saunders; 1994. p. 328-393.

11.Hobbins JC, Romero R, Grannum P, Berkowitz RL, Cullen M, Mahoney M. Antenatal diagnosis of renal anomalies with ultrasound. Obstructive uropathy. Am J Obstet Gynecol 1984; 148: 868-77.

12.Johnson MP, Bukowski PT, Reitleman C, Isada NB, Pryde PG, Evans MI. In utero surgical treatment of fetal obstructive uropathy: a new comprehensive approach to identify appropriate candidates for vesicoamniotic shunt therapy. Am J Obstet Gynecol 1994; 170: 1770-9.

13. Manning FA, Harrison MR, Rodeck C. Catheter shunts for fetal hydronephosis and hydrocephalus: report of the International Fetal Surgery Registry. N Engl J Med 1986; 315: 336-40.

14 - Phelan JP, Smith CV, Broussard P. Amniotic fluid volume assessment with the four-quadrant technique at 36-42 weeks' gestation. J Reprod Med 1987; 32:540-2. 